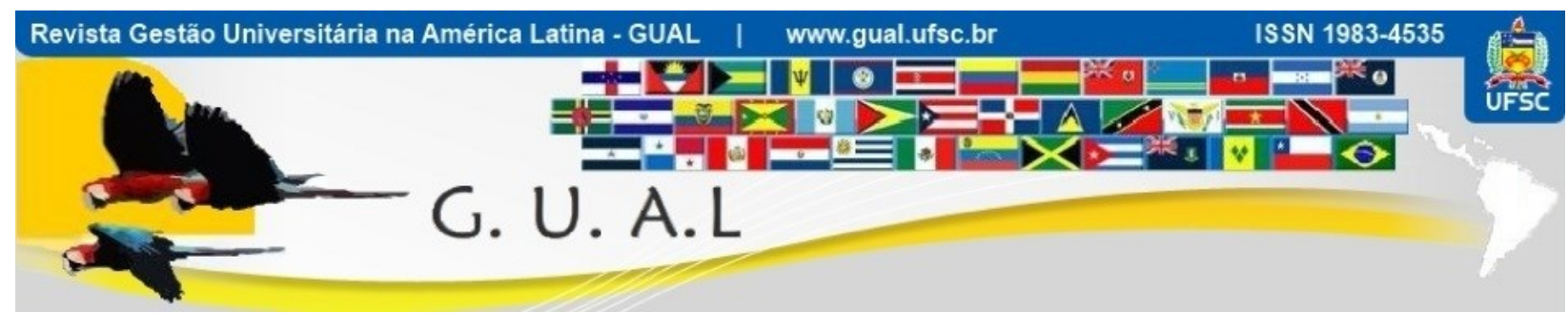

DOI: http://dx.doi.org/10.5007/1983-4535.2016v9n4p1

\title{
IMPLANTAÇÃO DO BALANCED SCORECARD (BSC) SOB O ENFOQUE DA VELHA ECONOMIA INSTITUCIONAL EM UMA INSTITUIÇÃO DE ENSINO SUPERIOR DO ESTADO DO RS
}

\section{IMPLEMENTATION OF THE BALANCED SCORECARD (BSC) IN FOCUS OLD INSTITUTIONAL ECONOMY IN AN INSTITUTION OF HIGHER EDUCATION OF RS STATE}

\author{
Maria Margarete Bacin Brizolla, Mestre \\ Universidade Regional do Noroeste do Estado do RS - UNIJUI \\ marga.brizolla@unijui.edu.br \\ Carlos Eduardo Facin Lavarda, Doutor \\ Universidade Federal de Santa Catarina - UFSC \\ elavarda@gmail.com \\ Roselaine Filipin, Mestre \\ Universidade Regional do Noroeste do Estado do RS - UNIJUI \\ roselaine.filipin@unijui.edu.br \\ Anelia Franceli Steinbrenner, Mestre \\ Universidade Regional do Noroeste do Estado do RS - UNIJUI \\ anelia.s@unijui.edu.br
}

Recebido em 04/dezembro/2015

Aprovado em 14/outubro/2016

Sistema de Avaliação: Double Blind Review

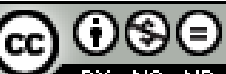

Esta obra está sob uma Licença Creative Commons Atribuição-Uso. 


\title{
RESUMO
}

O estudo teve como objetivo investigar como a adoção do BSC em um ambiente de ensino superior influência os conceitos institucionalizados e são convertidos em novos valores, hábitos e rotinas em uma em uma Instituição de Ensino Superior no Estado do RS. Para atender a este objetivo, foi realizada uma pesquisa descritiva, qualitativa, por meio de estudo de caso, por meio de entrevistas com os envolvidos no processo de implantação de BSC da instituição. Os resultados indicaram que a primeira conclusão observada é que a dimensão institucional é tão importante quanto a dimensão técnico-racional, na fase de concepção do modelo conceitual do sistema, não bastando planejar racionalmente os melhores conceitos, rotinas e regras para implantar um sistema de gestão, mas ter a base conceitual em sintonia com as diretrizes do campo institucional da organização. Percebe-se um processo consciente de identificação de princípios institucionais e de codificação do Balanced Scorecard especificando conceitos e rotinas coerentes com os princípios institucionais compartilhados pelos gestores institucionais. O estudo contribui para a compreensão mais adequada dos fenômenos reais de mudança e institucionalização de sistemas de gestão a partir de uma estrutura conceitual ficando evidenciado que a visão normativa é necessária, no sentido da adoção de melhores práticas de gestão, e a visão institucional tem sua importância no sentido de se assegurar a efetiva institucionalização do sistema idealizado.

Palavras-chave: Balanced scorecard. Teoria institucional. Instituição de ensino.

\begin{abstract}
The study aimed to investigate the adoption of the BSC at a higher influence learning environment institutionalized concepts and are converted into new values, habits and routines in a in a higher education institution in the state of RS. To meet this objective, a descriptive, qualitative was conducted through case study through interviews with those involved in the BSC implementation process of the institution. The results indicated that the first conclusion note is that the institutional dimension is as important as the technical-rational dimension in the conceptual model design phase of the system, simply not rationally plan the best concepts, routines and rules to implement a management system but have the conceptual basis in line with the guidelines of the institutional field of organization. It is noticed a conscious process of identifying institutional principles and the Balanced Scorecard coding specifying concepts and routines consistent with the institutional principles shared by institutional managers. The study contributes to a more adequate understanding of real phenomena of change and institutionalization of management systems from a conceptual framework becoming evident that the normative vision is needed towards the adoption of best management practices, and the institutional vision has its importance in order to ensure the effective institutionalization of the idealized system.
\end{abstract}

Keywords: Balanced scorecard. Institutional theory. Educational institution. 


\section{INTRODUÇÃO}

Considerado o ambiente complexo de inserção empresarial, é necessário a compreender o que as organizações desejam alcançar quando definem sua missão e as metas para tornarem-se bem sucedidas, onde um dos fatores a ser considerado é a importância de saber trabalhar com as mudanças contínuas do ambiente interno e externo (SENGE, 1991; SCHARMACH, 2010). No contexto das sucessivas mudanças, pressupõem-se a necessidade de implementar sistemas de acompanhamento e medição de desempenho, onde o ambiente de negócios da empresa tenha uma rápida adaptação as mudanças e a competitividade do mercado (ECCLES, 1991).

Uma ferramenta de gestão utilizada no ambiente empresarial capaz de acompanhar e medir o desempenho, é o Balanced Scorecard (BSC). Ele analisa por meio de indicadores de performance as perspectivas financeiras, as perspectivas dos clientes, os processos internos e a aprendizagem e crescimento com o objetivo de aumentar a lucratividade e a rentabilidade, reduzindo os custos e melhorando os processos (ZAGO et al., 2008).

Os estudos desenvolvidos em ambientes de serviços educacional, são em menor volume, muito embora se observe que o setor de serviços vem desempenhando um papel relevante na economia dos países desenvolvidos e em desenvolvimento (BRIGNALL, 1997). A adoção do BSC em uma intuição de ensino superior, utilizando como arcabouço conceitual a Velha Economia Institucional (Old Institutional Economics - OIE), pode subsidiar os envolvidos no processo de implantação da ferramenta com a introdução e internalização de novos conceitos, os quais ocorrem quando são institucionalizados, ou seja, quando são convertidos em novos valores, hábitos e rotinas dentro da organização (OLIVER, 1997).

Burns e Scapens (2000) utilizam os conceitos de hábitos, regras, rotinas e instituições para sugerir como as práticas podem tornar-se rotineiras no processo de implantação do BSC e, através do tempo, começar a fazer parte do conjunto das pressuposições e crenças inquestionáveis da organização (taken for granted).

Diversos autores no cenário nacional e internacional estudam a ferramenta Balanced Scorecard para a avaliação de desempenho das organizações. Dentro os autores internacionais merecem destaque: Martinsons, Davison e Tse (1999); Zago et al. (2008); Spessatto (2009); Lunkes et al. (2009); Scharmach (2010); Martins, Cruz e Corso (2011); Rocha e Lavarda (2011) e Machado (2013). 
Martinsons, Davison e Tse (1999) desenvolveram um BSC para os sistemas de informação (SI), que mede e avalia estas atividades a partir das perspectivas financeiras, do cliente, dos processos internos e do aprendizado e crescimento.

Zago et al (2008) realizaram um estudo de caso em uma distribuidora de bebidas de pequeno porte, aplicando o BSC na avaliação de desempenho da logística. Os resultados alcançados com esse novo modelo foram positivos, em que a empresa conseguiu traçar diretrizes que lhe proporcionasse vantagem competitiva, reduzindo os custos inerentes às atividades logísticas e proporcionando atitudes inovadoras e empreendedoras.

Spessatto (2009) analisou o processo de implantação do BSC nas 19 maiores empresas da região sul do Brasil. Demonstrando que o processo de implantação do BSC se mostrou relevante para alinhar a organização com a estratégia. A maior dificuldade enfrentada foi mobilizar todos a trabalharem com o modelo. E pode-se concluir que o processo de implantação do BSC ocorreu de acordo com o preconizado por Kaplan e Norton (1997).

Ainda se tratando da implantação do BSC, Lunkes et al. (2009) desenvolveram e implantaram o BSC em uma empresa de software, em que enfrentaram dificuldades na identificação dos indicadores relacionados com os objetivos estratégicos, mas conseguiram implantar o BSC em todos os setores da empresa: administrativo, comercial, marketing e técnico.

Martins, Cruz e Corso (2011) verificaram o impacto da implementação do BSC no desempenho financeiro de 20 empresas, analisando o "antes" e o "depois" da implantação. Os autores chegaram à conclusão de que a implantação do BSC não produz grandes diferenças no desempenho financeiro das empresas.

Rocha e Lavarda (2011), investigaram o uso do BSC em 71 artigos que apresentaram o BSC em estudos empíricos, e constataram que a cultura organizacional, o empenho e envolvimento da alta administração, a preparação e a motivação dos colaboradores são os principais fatores que facilitam ou inibem a implantação do BSC.

Com outra perspectiva, Machado (2013) analisou as taxas de utilização e a difusão do conhecimento sobre o BSC em 58 pequenas e médias empresas industriais portuguesas. Concluiu-se que poucas empresas utilizam o BSC e que a maioria dos gestores não conhece o método.

Lima, Soares e Lima (2012) realizaram uma análise da utilização do Balanced ScoreCard (BSC) em Instituições de Ensino Superior (IES), aonde foram analisadas vinte e 
cinco IES estrangeiras e mais dezessete IES nacionais, resultado foi que três perspectivas são mais recorrentes nas instituições do exterior: os processos internos, a financeira e os clientes. Já nas instituições nacionais, verificam-se quatro perspectivas em mais de metade das instituições analisadas: Processos Internos, Clientes, Aprendizado e Crescimento e Financeira. Assim, com a análise de quarenta e dois BSC de instituições de ensino, pode-se verificar a existência de quatro perspectivas mais comuns que são utilizadas para a mensuração de resultados.

Com esta abordagem, tem-se a seguinte questão problema: Como a adoção do BSC em um ambiente de ensino superior influência os conceitos institucionalizados e são convertidos em novos valores, hábitos e rotinas? Nesse sentido o objetivo deste estudo é investigar como a adoção do BSC em um ambiente de ensino superior influencia os conceitos institucionalizados e são convertidos em novos valores, hábitos e rotinas.

Scharmach (2010) contribuiu com os estudos em gestão estratégica pelo modelo BSC em IES, demonstrando as possibilidades e limitações de sua aplicação. Os gestores da IES onde foi aplicado o estudo, consideraram importante e necessária a utilização de um modelo que delineie estratégias e faça avaliações de desempenho para a competitividade da instituição. Eles consideraram oportuna a aplicação do BSC desde que haja adaptações com as características peculiares desse tipo de organização. Os resultados da pesquisa foram satisfatórios, revelando que a universidade possui mais possibilidades que limitações para a aplicação do BSC.

Este trabalho demonstra sua relevância ao analisar o processo de implantação do BSC no contexto de uma instituição de ensino superior, a partir do conhecimento e internalização dos conceitos, buscando identificar os possíveis obstáculos na utilização e novas linhas de pesquisa empírica dentro do tema proposto.

\section{REFERÊNCIAL TEÓRICO}

No referencial teórico são abordados aspectos conceituais sobre a avaliação de desempenho e o Balanced Scorecard, suas medidas de desempenho e indicadores de performance. 


\subsection{AVALIAÇÃO DE DESEMPENHO E BALANCED SCORECARD (BSC)}

As empresas buscam, por meio da avaliação de desempenho, monitorar suas funções com o objetivo de verificar a repercussão de suas ações em relação aos clientes, com a necessidade de buscar formas que as diferem de seus concorrentes (ZAGO et al., 2008). De acordo com Fereira e Otley (2009) a medição de desempenho empresarial constitui-se em um instrumento para gestão das organizações, em razão de possibilitar um monitoramento contínuo da informação. É como se fosse um guia que pode ajudar a mover a organização de onde ela está para onde ela deveria estar.

As medidas de desempenho fornecem um feedback gerencial que é utilizado para a tomada de decisão, facilitando a identificação do estágio atual e um entendimento para a evolução futura (YUEN, 2006). Destaca-se ainda a existência de duas modalidades de medidas: as tangíveis e as intangíveis. As tangíveis são medidas diretas, como o total dos custos e o número de entregas. As intangíveis são indiretas, como atitudes, capacidades dos serviços, goodwill e reputação (KAPLAN; NORTON, 2004; YUEN, 2006).

Acredita-se que a construção de indicadores de desempenho de forma adequada está associada ao sucesso de um sistema de avaliação de desempenho organizacional. Nesse sentido, algumas características, e propriedades necessitam que sejam percebidas na construção desses indicadores, tais características dizem respeito aos indicadores desempenho, os quais devem respeitar os demais objetivos da organização, sendo importante o alinhamento entre estes os demais objetivos da organização permite ao gestor decidir acertadamente (MERCHANT, 2006). Nesse sentido entende-se que o indicador deve ser controlável pelo gestor, caso isso não ocorra o indicador perde o poder motivacional (DUTRA, 2004).

Olson e Slater (2002) destacam três elementos fundamentais para os sistemas de informações, avaliação e controle: estabelecimento dos padrões de desempenho; a mensuração do desempenho frente aos padrões estabelecidos e a tomada de ações corretivas, caso os padrões não sejam alcançados. Um sistema de mensuração de desempenho deve ser compreensível, equilibrado, completo e refletir melhorias (SCHIMIDT; KIEMELE; BERDINE, 1999). Trata de um sistema de informações que os gestores fazem uso para rastrear a implementação da estratégia do negócio, comparando os resultados reais aos objetivos e metas estratégicas (SIMONS, 2000). 
Nesse sentido o Balanced Scorecard (BSC) apresenta-se como um método de avaliação do desempenho organizacional, desenvolvido por Kaplan e Norton por meio de pesquisas realizadas em organizações. Os autores partiram da ideia de que os ativos intelectuais deveriam ser mensurados, pois a exclusiva dependência da medida de um sistema financeiro é insuficiente (KAPLAN; NORTON, 2001).

A diferença do BSC é que os indicadores de desempenho financeiro são complementados com os indicadores operacionais referindo-se aos processos internos, à satisfação do cliente e às ações que impulsionam o desempenho financeiro futuro da empresa (KAPLAN; NORTON, 2001). O BSC visa auxiliar a organização na definição de um conjunto de medidas que reflete os objetivos organizacionais e avaliar o seu desempenho de forma adequada, equilibrando medidas financeiras e não-financeiras (KENNERLEY; NEELY, 2002).

Kaplan e Norton (2001, p. 119) afirmam que o BSC é "um conjunto de indicadores que proporciona aos gestores uma visão rápida, embora abrangente, de toda a empresa". É um conjunto de medidas previamente selecionadas provenientes da estratégia organizacional, é um instrumento utilizado para avaliar se os objetivos estratégicos foram alcançados (NIVEN, 2002).

O BSC é um importante instrumento de gestão, pois apresenta uma visão voltada para a estratégia organizacional, apresentando um conjunto de medidas de desempenho que objetiva um sistema de gestão eficiente (KAPLAN; NORTON, 1997). De acordo com os autores, o BSC possui quatro perspectivas que permite um equilíbrio entre os objetivos de curto e longo prazo: perspectiva financeira; do cliente; dos processos internos; do aprendizado e do crescimento.

A perspectiva financeira considera aspectos para medir a repercussão econômica de ações passadas, estando vinculados à estratégia da organização, definindo o desempenho esperado. Deve demonstrar que as escolhas estratégicas estão gerando valor econômico e de mercado para a empresa, e de riqueza para os stakeholders (KAPLAN; NORTON, 1997; HERRERO FILHO, 2005).

Prieto et al (2006, p. 83) afirma que "os objetivos e medidas financeiras desempenham um papel duplo: definem o desempenho financeiro esperado da estratégia e servem de meta principal para a definição dos objetivos e medidas das outras perspectivas do scorecard". Os objetivos financeiros precisam estar relacionados com outras metas que complementam as 
demais perspectivas do BSC, proporcionando assim oportunidades de aperfeiçoamento para as organizações (SPESSATTO, 2009).

A perspectiva do cliente visa demonstrar se as escolhas estratégicas implementadas pela organização estão contribuindo para aumentar o valor percebido pelos clientes em relação aos serviços prestados, aos produtos vendidos e a imagem de marca, pois cumprir a necessidade do cliente é essencial para qualquer empresa (HERRERO FILHO, 2005; ROCHA, 2002). Nesta perspectiva, o BSC "permite que os executivos identifiquem os seguimentos de clientes e mercados nos quais a unidade de negócios competirá e as medidas do desempenho da unidade nesses segmentos-alvo" (KAPLAN; NORTON, 1997, p. 25). Além disso, o BSC identifica os indicadores que evidenciam os resultados desta expectativa, relacionando-se com a estratégia estabelecida pela organização para ir ao mercado (ROCHA, 2002).

Kaplan e Norton (1997, p. 25) afirmam que "as medidas dos processos internos estão voltadas para os processos internos que terão maior impacto na satisfação do cliente e na consecução dos objetivos financeiros da empresa". As organizações devem buscar avaliar o grau de inovação em seus processos de gestão e o nível de qualidade de suas operações (FERNANDES; BERTON, 2005). A perspectiva dos processos internos visa verificar se o plano estratégico para gerar valor econômico para o cliente, elevando o valor de mercado da organização e a riqueza para os stakeholders está sendo implementado com sucesso, identificando e medindo suas habilidades essenciais e tecnológicas necessárias (HERRERO FILHO, 2005).

A perspectiva de aprendizado e crescimento do BSC identifica a infraestrutura que a empresa deve construir para criar melhorias e crescimento no longo prazo, são considerados vetores de resultados excelentes das três primeiras perspectivas do scorecard (KAPLAN; NORTON, 1997). Segundo Fernandes e Berton (2005) essa perspectiva busca atingir a manutenção do capital intelectual com um alto nível de motivação, satisfação interna e produtividade. Além de ser usada para avaliar o grau de criatividade e alinhamento estratégico dos colaboradores.

Para atingir suas metas de longo prazo para clientes e processos internos, é necessário melhorar as tecnologias e capacidades atuais, pois as fontes principais do aprendizado e crescimento são os sistemas e procedimentos e as pessoas envolvidas, que são necessárias 
para atingir um desempenho inovador (KAPLAN; NORTON, 1997). Essa perspectiva é considerada a base de sustentação de toda a organização.

As quatro perspectivas apresentadas acima buscam o alinhamento dos objetivos estratégicos das organizações com as metas e estão interligadas por relações de causa e efeito, visando auxiliar numa gestão estratégica integrada, ajudando os gestores na tomada de decisão (LUNKES et al., 2009).

De maneira integrada, o BSC demonstra as habilidades, o conhecimento e os sistemas que os colaboradores precisarão (seu aprendizado e crescimento), para construir e inovar nas capacidades estratégicas corretas e eficientes (processos internos) que resultarão em valores específicos ao mercado (clientes), os quais, consequentemente, irão proporcionar um aumento do valor ao acionista (financeiro) (PIETRO et al., 2006). As quatro perspectivas do BSC devem ser considerados um modelo, e não algo a ser seguido forçadamente, pois não existe um modelo padrão, em que as quatro perspectivas sejam necessárias e suficientes. Mas, elas tem-se revelado adequadas em diversas organizações e setores (KAPLAN; NORTON, 1997).

Kaplan e Norton (2004) discorrem que o BSC introduziu sistemas de medidas de performance para quantificar ativos tangíveis e intangíveis. Os processos de avaliação de desempenho são um conjunto de indicadores e relatórios utilizados pela organização para avaliar o seu desempenho (MIRANDA; SILVA, 2002).

\subsection{TEORIA INSTITUCIONAL}

A teoria institucional considera que o comportamento individual é determinado por padrões criados, os quais são compartilhados na interação e internalizados como normas e regras objetivas, cristalizadas na organização como concepções legitimadas sobre o modo mais eficaz de funcionamento das organizações. O interesse em estudar a relação da teoria institucional com as ciências sociais tem aumentado, podendo ser observado três enfoques dessa teoria dessa teoria na literatura contábil: (i) nova sociologia institucional (NIS - new institutional sociology), (ii) nova economia institucional (NIE - new institutional economics) e (iii) velha economia institucional (OIE - old institutional economics) (FONSECA; MACHADO DA SILVA, 2002).

Nesse estudo aborda-se a Teoria Institucional na ótica Old Intitucional Economics para determinar que a instituição é o principal objeto de análise, e não o comportamento racional e maximizador dos indivíduos tomadores de decisões (REIS, 2008). O conjunto de 
abordagens da Velha Economia Institucional fornece arcabouço intelectual ao entendimento dos sistemas de contabilidade gerencial. Burns (2000) salienta que a Velha Economia Institucional focaliza no estudo das mudanças nas rotinas dos sistemas de gestão. Além disso, o foco é direcionado aos fatores internos, ajustando o comportamento dos indivíduos a adoção de novos conceitos, vislumbrando uma legitimação interna.

Assim, a instituição é o principal objeto de análise na ótica da Velha Economia Institucional. Burns e Scapens (2000) definem a instituição como pressupostos compartilhados e aceitos de forma inquestionável, pelos quais identificam categorias de atores, suas atividades e relacionamentos apropriados. Burns (2000) salienta que a Velha Economia Institucional considera instituição como uma forma de pensar ou agir em algo que prevalece e permanece, o qual está inserido nos hábitos de um grupo.

A OIE oferece um novo arcabouço conceitual que explica a estabilidade e a mudança nas práticas contábeis gerenciais, introduzindo na análise as dimensões sociais, políticas e culturais, no sentido de entender a estabilidade e a mudança no desenvolvimento das práticas contábeis, rejeitando os pressupostos de racionalidade econômica e equilíbrio de mercado. A organização é o objeto de análise e não os tomadores de decisões individuais. Está centrado na mudança econômica, mais que no equilíbrio econômico da teoria neoclássica (RUSSO et al., 2012).

Nesse sentido, a abordagem institucional é apresentada como uma estrutura válida para o entendimento das práticas de contabilidade gerencial como rotinas institucionalizadas (HUMPHREY; SCAPENS, 1996; SCAPENS; ROBERTS, 1993). Esta perspectiva teórica estuda como e porquê, determinados comportamentos ou estruturas surgem, se mantêm ou mudam ao longo do tempo (MOLL; BURNS; MAJOR, 2006).

Para Scapens (1990), o comportamento humano não pode ser reduzida às escolhas $\mathrm{e}$ decisões de indivíduos em particulares, as circunstâncias são advindas em parte da ação individual, onde os indivíduos são moldados pelas circunstâncias, incluindo a sua interação com outros indivíduos e os fatores que explicam a conduta humana não podem ser reduzidos a priori a nenhuma hipótese geral, abstrata ou não-histórica. Podendo os pressuposto iniciais a respeito da racionalidade, eliminar a necessidade do conhecimento da conduta humana, fundada em seu contexto institucional e cultural.

Burns e Scapens (2000) sustentam a contabilidade gerencial como uma instituição, oferecendo conceitos como hábitos, rotinas e instituições, os quais permitem sugerir que as 
práticas contábeis se tornem rotineiras passando a fazer parte de um conjunto de crenças inquestionáveis. Assim, a Velha Economia Institucional busca entender como os hábitos e as regras são transmitidas, replicadas e estruturadas em torno das atividades organizacionais ao longo do tempo, através da codificação, incorporação, reprodução e institucionalização (HODGSON, 1998).

De acordo com a Velha Economia Institucional, ao adotar a ferramenta Balanced Scorecard leva-se em conta o contexto organizacional interno e externo, assumindo a existência de lacunas entre as delimitações associadas ao BSC e as práticas institucionalizadas na organização, sendo assim, as ações dos indivíduos são influenciadas por regras e rotinas e não pelos princípios prescritos na literatura do BSC. Nesse sentido a adoção dessa ferramenta pode oferecer resistência e conflito, se as mudanças desafiarem as rotinas e não forem consistentes com as instituições atuais (SIMÕES; RODRIGUES, 2012).

A dinâmica do modelo de Burns e Scapens (2000) demonstra a ligação entre o campo institucional e o campo da ação. Primeiramente, o campo institucional codifica princípios institucionais em regras e rotinas e, na sequência, os atores, através de suas ações e interações com o campo da ação, incorporam as regras e rotinas que codificam os princípios institucionais. Esse modelo apresenta quatro processos: codificação; incorporação; reprodução; e institucionalização.

A codificação requer que os princípios institucionais idealizados sejam codificados, detalhados e especificados em rotinas e regras, este processo é orientado pelo conjunto de princípios desejados e impactado pelas rotinas e regras existentes no processo de concepção de um novo sistema de contabilidade gerencial, sendo necessário entender as premissas de gestão e os modelos decisórios dos gestores, identificando o campo institucional da organização. Se completando, com a definição de premissas, especificação de conceitos, definição de critérios analíticos de mensuração e de identificação de resultados com objetos de gestão (BURNS; SCAPENS, 2000).

A incorporação contempla os envolvidos incorporando as rotinas e regras, as quais codificam os princípios institucionais desejados pela organização, podendo envolver escolha consciente, mas normalmente é resultado de um monitoramento reflexivo e da aplicação do conhecimento tácito a respeito de como as coisas devem ser feitas. A incorporação de novas rotinas e regras pode estar sujeita a resistências, especialmente se as novas rotinas e regras 
desafiarem os significados e valores existentes e se os envolvidos tiverem poder suficiente poder para interferir no processo (BURNS; SCAPENS, 2000).

A reprodução ocorre à medida que o comportamento repetitivo conduz à reprodução de rotinas. Burns e Scapens (2000) observam que pode envolver escolha consciente ou inconsciente. A ocorrência da escolha consciente é provável quando os envolvidos reúnem elementos que permitam coletivamente questionar as rotinas e regras existentes.

Por fim a institucionalização de regras e de rotinas reproduzidas por meio de comportamentos repetitivos dos envolvidos individuais pressupõe que os padrões de comportamento atuais sejam desassociados de suas circunstâncias históricas. (BURNS; SCAPENS, 2000; SIMÕES; RODRIGUES, 2012).

Desta forma, a implementação de novas formas de gestão pode ser caracterizada como institucionalizada, quando se torna amplamente aceita na organização e quando se transforma em um controle de gestão (HUMPHREY; SCAPENS, 1996; SCAPEN; ROBERTS, 1993; BURNS; SCAPENS, 2000).

\section{METODOLOGIA}

O presente estudo possui característica descritiva que decorre do fato de que o estudo tem como finalidade investigar como a adoção do BSC em um ambiente de ensino superior, utilizando a Velha Economia Institucional (Old Institutional Economics - OIE), influencia os conceitos institucionalizados e são convertidos em novos valores, hábitos e rotinas em uma Instituição de Ensino Superior no Estado do RS, descrevendo essa influência. Segundo Cervo e Bervian (2002), a pesquisa descritiva expõe características de determinada população ou de determinado fenômeno.

Em relação à abordagem, essa pesquisa se classifica como qualitativa, pois os dados pesquisados serão analisados com certa profundidade, e não serão utilizados instrumentos estatísticos tanto na coleta quanto no tratamento dos dados. Segundo Richardson (1999, p. 80), a pesquisa qualitativa tem a capacidade de "descrever a complexidade de determinado problema, analisar a interação de certas variáveis, compreender e classificar processos dinâmicos vividos por grupos sociais".

Utiliza-se como procedimento de pesquisa um estudo de caso junto à Instituição de Ensino Superior no Estado do RS. Na concepção de Yin (2010, p. 39) “o estudo de caso é uma investigação empírica que investiga um fenômeno contemporâneo em profundidade e em 
seu contexto de vida real, especialmente quando os limites entre o fenômeno e o contexto não são claramente evidentes".

A investigação foi focada especificamente na modelagem do sistema que, sob o prisma da estrutura conceitual de Burns e Scapens (2000), corresponde à fase da codificação das regras e rotinas a partir dos princípios do campo institucional da organização. $\mathrm{O}$ desenvolvimento da pesquisa ocorreu na fase em que a instituição estava validando o modelo conceitual do BSC e iniciando sua implantação (implantando em um setor teste).

Os dados foram obtidos por meio de entrevistas e de análise de documentos. As entrevistas foram conduzidas junto ao gestor do processo de mudança, gestores da área de Controladoria, responsável pela implementação do BSC. Foram realizadas cinco entrevistas de aproximadamente uma hora no período de 28 de maio a 11 de junho de 2013 com o responsável pela implantação do sistema; com 4 colaboradores internos envolvidos no processo; e com o responsável pela consultoria externa. Tais entrevistas tiveram o objetivo de conhecer: a) os antecedentes ao processo de mudança; b) as características organizacionais e operacionais da instituição; e c) o processo de modelagem utilizado para a concepção do BSC.

Com o objetivo de conferir um maior grau de confiabilidade às informações fornecidas pelo entrevistado, realizou-se um processo de triangulação, por meio de análise documental, pesquisando-se: a) documentos oficiais da instituição, relativos à estrutura de organização, funcionamento de processos e atividades e relatórios financeiros; b) relatório, atas come depoimentos dos executivos das diversas áreas da instituição de ensino superior sobre necessidades, expectativas e percepção quanto à implantação do novo sistema (BSC); e c) relatório da modelagem $\mathrm{BSC}$ estudado.

\section{DESCRIÇÃO E ANÁLISE DOS RESULTADOS}

A descrição e análise dos resultados objetivam demostrar como foi atendido o objetivo de estudo proposto e também apresentar a resposta à questão de investigação, nesse sentido apresentam-se na sequencia cinco sessões que tem o intuito de atender a este propósito.

\subsection{CARACTERÍSTICAS DO NEGÓCIO}

A entidade estudada é uma instituição de ensino superior do RS inserida em uma importante região do Estado. Esta instituição ocupa a primeira posição dentre as quatro 
maiores instituições classificadas pelo critério de número de alunos, com aproximadamente 9.000 alunos.

A instituição é estruturada em três unidades geradoras de receitas: a) a graduação; b) a pós graduação; e c) a educação continuada. A unidade de negócio com maior representatividade na composição das receitas é a graduação.

As unidades de apoio são responsáveis por diversas atividades, sendo as mais representativas: Engenharia e Patrimônio, Marketing, Recursos Humanos, Jurídico, Controladoria, Contabilidade e Auditoria Interna, responsáveis por $91 \%$ dos custos da organização. As unidades de produção dos serviços correspondem à prestação de serviço de educação na graduação, sendo professores o de destaque, embora diversas outros como Tecnologia de Informação façam parte dessa classificação. O custo direto desses órgãos de produção corresponde a $86 \%$ do total dos custos da instituição.

\subsection{ANTECEDENTES AO PROCESSO DE MUDANÇA}

Questões que podem levar a uma reorganização estrutural nas instituições são as necessidades recorrentes de informações precisas em tempo hábeis para a tomada de decisão. No estudo proposto este foi o marco para se pensar uma ferramenta de gestão capaz de ao mesmo tempo apresentar medidas financeiras e não financeiras. Nesse sentido, ambientes diferentes ao longo do tempo requerem o entendimento e incorporação de novos conceitos. As mudanças externas influenciam mudanças nos sistemas de gestão e as principais características do atual ambiente social e institucional constituem-se em fatores indutores de processos de mudanças (BAINES; LANGFIELD-SMITH, 2003).

Nesta seção é realizada uma retrospectiva das circunstâncias que levaram à implantação do BSC na instituição objeto do estudo de caso. A instituição investiu recursos materiais e humanos próprios por longo período de tempo em consultoria para conceber e implantar um modelo conceitual de sistema de orçamentário e de custos, que tinha por objetivo satisfazer a duas necessidades: a) apuração e gestão de custos e resultados; e b) acompanhar e avaliar o orçado e realizado.

Os custos são apurados baseando-se na filosofia do Custeio Variável, alocando os custos por contas e por centros de custos. Aos serviços foram apropriados os custos variáveis (todos diretos) e alocados apenas os indiretos relativos ao processamento de documentos (Tecnologia de Informação). Os representados pela unidade de negócio Corporação, aí 
incluídos os de administração e reitoria, não eram repassados aos produtos nem aos clientes. Nesse sentido, Crepaldi (2012) acrescenta que o custo dos produtos vendidos, os produtos em elaboração e os produtos acabados só conterão custos variáveis. O custeio variável/direto de acordo com Horngren, Sunden e Stratton (2004) refere-se a um sistema de custeio com ênfase no custo do produto que considera os custos variáveis e os custos fixos são excluídos dos custos do produto, sendo considerados gastos do exercício, reconhecidos na demonstração do resultado do exercício.

Os respondentes destacaram a importância do orçamento para a instituição, colocando que por meio do orçamento são projetados e analisados os resultados, comparados indicadores de desempenho para a partir de então, redimensionar os investimentos ou corrigir as possíveis distorções. O orçamento é o plano financeiro que põe em prática a estratégia da empresa para determinado período, não é uma simples ferramenta utilizada para as projeções de resultados, se embasa no comprometimento dos gestores com o alcance das metas definidas (FREZATTI, 2007).

Investiu-se também, recursos materiais e humanos, incorrendo em gastos relevantes com formação de pessoal que não trouxeram os retornos esperados com produção científica, isto é, à medida que a formação ocorria havia uma rotação de pessoal, troca por instituições federais.

\subsection{A IDENTIFICAÇÃO DO CAMPO INSTITUCIONAL}

A teoria institucional está centrada nas organizações e nos indivíduos que são parte integrante de elementos culturais que definem o modo como o mundo é e como deveria ser, isto é, de uma rede de valores, normas, regras, crenças e pressuposições aceitas sem questionamentos. Esses elementos detalham e especificam as formas de procedimentos a serem seguidos pelos membros de uma organização em determinado momento. Sendo assim, o campo institucional representa uma estrutura de regras e tipificações, derivadas de uma história acumulada de ações e interações com influências culturais no processo de tomada de decisões e nas estruturas formais, representam restrições no processo de escolhas que os indivíduos e os grupos gostariam de fazer, embora essas restrições estejam o tempo todo abertas para modificações (BARLEY; TOLBERT, 1997).

As rotinas e regras concretas são materializadas no campo institucional e estão marcadas mais enfaticamente os valores e pressuposições aceitas. Entende-se como 
apropriado que a primeira etapa na aplicação da teoria seja uma análise do campo institucional com a identificação do conjunto inicial de rotinas e regras que caracterizam o controle gerencial da instituição (SOIN; SEAL; CULLEN, 2002).

Conforme informações obtidas nas entrevistas, o processo de implantação do BSC foi desenvolvido pela equipe do projeto em três etapas. Na primeira, foi realizada pesquisa interna com 11 gestores da instituição para identificar as principais necessidades informativas desses gestores, bem como sua visão sobre os objetivos e as dimensões relevantes que deveria possuir o BSC. Foram consultados membros do conselho de gestão, gestores das áreas comerciais e gestores das áreas de apoio. Para a coleta de dados foram aplicados questionários com questões abertas. Na segunda etapa, realizou-se um benchmarking, utilizando-se de modelos já aplicados por consultores externos em outras instituições de ensino superior de porte e características similares às deste estudo, procurando conhecer os modelos e funcionamento de BSC utilizados por elas, o tempo médio de implantação, os fatores críticos de sucesso e as armadilhas a serem evitadas. Na terceira, o modelo foi discutido com uma equipe de consultores externos.

Com base no aprendizado obtido com as três etapas descritas acima, a equipe do projeto, liderada pela área de Controladoria, iniciou o delineamento das premissas, dos princípios e das diretrizes que deveriam nortear a construção do modelo conceitual do BSC para a instituição em questão.

Verificou-se inicialmente que, embora a pesquisa interna revelasse que as medidas financeiras e não financeiras eram bem aceitas pelos gestores da instituição, não havia consenso quanto à abrangência do processo de geração e utilização desses indicadores, devendo ser utilizada uma abordagem mais clara quanto ao entendimento conceitual e sua utilização. Havia consenso, no entanto, de que, independentemente da decisão sobre o grau de utilização, esses indicadores deveriam ser realizados no nível do gestor que tivesse autonomia para implementar medidas corretivas.

A pesquisa interna revelou ainda que a principal preocupação dos executivos relacionava-se com a previsibilidade dos indicadores; assim, o BSC deveria ter a característica de proporcionar uma antevisão dos valores atribuídos, evitando-se surpresas. Essa preocupação foi quase unânime entre os pesquisados, os quais deixaram claro que a previsibilidade dos indicadores, na sua visão, era mais importante do que a exatidão dos números apurados e do que a acurácia do BSC. 
Com base na pesquisa realizada foram definidos os seguintes princípios que deveriam nortear todo o processo de concepção do BSC: a) o sistema deveria prover efetivo suporte ao processo de gestão e ser consistente com o modelo de estrutura organizacional; b) o sistema deveria refletir a natureza dos processos do negócio; c) o sistema deveria possibilitar total transparência do processo de formação dos indicadores; d) as informações geradas pelo sistema deveriam dar suporte ao processo de medição e acompanhamento dos indicadores; e) as informações geradas pelo BSC deveriam dar suporte ao processo de avaliação de desempenho na organização; e f) o BSC deveria dar segurança aos gestores quanto à previsibilidade dos indicadores.

\subsection{O PROCESSO DE CODIFICAÇÃO}

Do ponto de vista de desenvolvimento de sistemas, o processo de codificação corresponde à fase de modelagem do sistema, ou seja, à especificação do modelo conceitual e lógico que deverá orientar as soluções de tecnologia de informação. A abordagem normativa em contabilidade gerencial menciona que o modelo deve ser especificado de forma a gerar informações eficazes para o processo de tomada de decisões gerenciais racionais (BURNS; SCAPENS, 2000). Nesse contexto, Kaplan (1990) relata que as empresas estão desenvolvendo novos sistemas, dentre eles o BSC para produzir informações úteis para o controle operacional e para a análise da rentabilidade de produtos e serviços, linhas de produtos e clientes. O desenho do sistema de gestão, como é o caso do BSC, deve ser efetuado considerando a missão e estratégia da organização e o tipo de processo do serviço (BRIGNALL,1997). Martinsons, Davison e Tse (1999) desenvolveram um BSC que mede e avalia estas atividades a partir das perspectivas financeiras, do cliente, dos processos internos e do aprendizado e crescimento.

À luz da abordagem institucional, o processo de codificação requer que os princípios institucionais sejam codificados, isto é, detalhados e especificados em rotinas e regras. Burns e Scapens (2000) observam que o processo de codificação é influenciado pelas rotinas e instituições vigentes, pelas intenções (racionais) dos agentes de mudanças e por outras instituições fora da organização. As novas regras e rotinas codificadas são influenciadas pelas regras e rotinas vigentes e nesse sentido elas terão mais chance de ser implementadas à medida que forem mais consistentes com as regras e rotinas amplamente aceitas no âmbito da 
organização. Por outro lado, o processo de codificação também é fortemente influenciado pela intenção consciente e racional dos agentes de mudança.

Diante disso, destaca-se os conteúdos das rotinas e regras que não se conformam com os valores do grupo social (se deseja eliminar), e os elementos que reflitam da melhor forma possível preocupações com eficiência econômica, indicadores financeiros e não financeiros e tomada de decisões gerenciais (se deseja adicionar). Os agentes de mudança também serão influenciados por outras instituições normalmente fora da organização, tais como práticas utilizadas em empresas pesquisadas (melhores práticas), opiniões de consultores, recomendações de associações profissionais e ainda por grupos que exerçam algum tipo de poder sobre o time do projeto (SOIN; SEAL; CULLEN, 2002).

Com base nos princípios e diretrizes estabelecidos em função a) das informações obtidas com a pesquisa interna realizada junto aos executivos, b) do benchmarking realizado e c) das reuniões com os consultores externos, a equipe da Controladoria, condutora do projeto, definiu os conceitos de mensuração a serem utilizados BSC. Do ponto de vista do modelo de Burns e Scapens (2000), esta etapa corresponde ao processo de codificação, em que os princípios institucionais são codificados em regras e rotinas. No estudo em questão pode-se observar a partir das entrevistas que os envolvidos no processo tem clareza sobre o conceito do BSC e suas quatro perspectivas, tal conhecimento segundo os mesmos foram obtidos a partir de rodadas de discussões e também estudando a literatura pertinente ao tema.

Quando perguntado se o entrevistado saberia dizer de que se tratava a ferramenta BSC e qual sua utilidade este respondeu:

$\mathrm{O}$ BSC se refere a indicadores que permitem aos gerentes ter uma ampla e rápida, da instituição. Esses indicadores são previamente definidos e são originados das estratégias, das discussões e servem para avaliar se os objetivos estratégicos foram ou estão sendo atingidos (ENTREVISTADO 01).

Quando perguntado a outro entrevistado sobre as quatro perspectivas do BSC o que significavam, obteve-se a seguinte resposta:

A perspectiva financeira considera para medir, os aspectos econômicos de ações passadas, está vinculado à estratégia da instituição, definindo assim o desempenho esperado.

A perspectiva do cliente demonstra se as estratégias implementadas pela instituição estão contribuindo para melhorar o valor percebido pelos clientes (alunos) em relação aos serviços prestados e a imagem de marca.

A perspectiva dos processos internos verifica se o plano estratégico que gera valor econômico para o cliente, elevando o valor de mercado da instituição está sendo 
implementado com sucesso, identificando e medindo suas habilidades essenciais e tecnológicas.

A perspectiva de aprendizado e crescimento do BSC identifica a infraestrutura que a empresa precisa para criar melhorias e crescimento no longo prazo, buscando atingir a manutenção do capital intelectual com um alto nível de motivação, satisfação interna e produtividade.

\subsection{DISCUSSÃO DO CASO}

Conforme mencionado anteriormente, a modelagem, também denominada concepção do modelo conceitual de um BSC, equivale ao primeiro processo do modelo de Burns e Scapens (2000) denominado codificação. O processo de codificação se desenvolve inicialmente a partir da identificação dos princípios institucionais idealizados. Soin, Seal e Cullen (2002) observam que o primeiro passo na aplicação do modelo de Burns e Scapens (2000) é a análise do campo institucional vigente (institutional realm). Após essa fase, desenvolve-se a codificação propriamente dita, onde os princípios institucionais idealizados são codificados, detalhados e especificados em rotinas e regras.

A identificação dos princípios institucionais é fundamental para o sucesso da concepção do sistema e deve ser efetuada de forma consciente pela equipe do projeto, uma vez que esses princípios idealizados serão especificados em conceitos, sendo materializados em software, em tecnologia de informação.

No caso em estudo, pode-se perceber que havia um sistema orçamentário institucionalizado e os custos eram calculados utilizado o custeio variável e planilhas eletrônicas não havendo um sistema institucionalizado. As medidas não financeiras eram obtidas a partir do sistema integrado eletronicamente (SIE), no entanto muitos indicadores financeiros e não financeiros eram obtidos manualmente, o que propiciou a busca por uma ferramenta de gestão que atendesse esta demanda. Conforme pode ser observado em Burns e Scapens (2000), um novo sistema gestão (rotinas e regras codificados) que apresente contradições em relação às rotinas e regras existentes (campo institucional vigente) pode trazer conflito e resistência, levando ao insucesso na implementação do novo sistema.

As informações coletadas neste estudo demonstram que, no desenvolvimento do novo sistema, a equipe do projeto estava muito consciente no sentido de captar as opiniões dos gestores da instituição, desenvolvendo pesquisa com mais de 11 gestores institucionais. Os dados do estudo demonstram que a equipe do projeto identificou os princípios institucionais idealizados pelos gestores relativos ao BSC. 
O processo de codificação (especificação de conceitos), conforme se pode observar pelas informações da seção 4.4 deste trabalho, foi desenvolvido pela equipe do projeto de acordo com a orientação de atender efetivamente as visões dos gestores da instituição. Nesse sentido, esse processo de codificação, que envolveu a especificação de conceitos e o detalhamento de regras e rotinas, se deu de forma coerente com os valores do campo institucional da instituição de ensino superior. Um aspecto de destaque nesse processo é que não ocorreu uma submissão cega às opiniões dos gestores. A preocupação com o aspecto institucional caminhou em paralelo com a dimensão racional-normativa, no sentido da definição dos indicadores financeiros e não financeiros a partir das quatro perspectivas.

\section{CONSIDERAÇÕES FINAIS}

O objetivo desta pesquisa foi investigar como a adoção do BSC no ambiente de uma instituição de ensino superior, utilizando a Velha Economia Institucional (Old Institutional Economics - OIE), influencia os conceitos institucionalizados e são convertidos em novos valores, hábitos e rotinas em uma em uma Instituição de Ensino Superior no Estado do RS, descrevendo como ocorreu o processo de codificação das regras, rotinas e conceitos do sistema a partir da identificação dos princípios institucionais da organização. $\mathrm{O}$ arcabouço conceitual utilizado no estudo foi o modelo de Burns e Scapens (2000).

A primeira conclusão observada no estudo, em linha com as diretrizes do arcabouço teórico, é que a dimensão institucional é tão importante quanto a dimensão técnico-racional, na fase de concepção do modelo conceitual do sistema. Isso significa dizer que não basta planejar racionalmente os melhores conceitos, rotinas e regras para implantar um sistema de gestão, mas também que essa base conceitual planejada esteja em sintonia com as diretrizes do campo institucional da organização. Essa sintonia somente será possível através da identificação das crenças e valores que permeia a organização e que faz parte do denominado campo institucional.

No caso da instituição objeto de estudo, pode-se perceber que houve um processo consciente de identificação de princípios institucionais e que o processo de codificação do BSC especificou conceitos e rotinas coerentes com os princípios institucionais compartilhados pelos gestores institucionais.

Considerando-se as premissas da teoria institucional, é possível afirmar que, independentemente dos aspectos conceituais técnicos, o sistema modelado tem grande 
probabilidade de ser institucionalizado, ou seja, de se converter em um instrumento efetivamente aceito na instituição. Essa probabilidade de sucesso de institucionalização está associada a lógica de especificação dos conceitos do BSC. A lógica institucional indica que a fase de codificação das novas regras e rotinas deve ser orientada pelas crenças e valores presentes no campo institucional vigente na empresa.

Neste caso percebe-se que houve um processo consciente de identificação de princípios institucionais e que no processo de codificação foram definidos e detalhados conceitos e rotinas coerentes com os princípios institucionais da instituição.

Apesar das limitações metodológicas, o estudo traz contribuições efetivas, das quais a principal delas é proporcionar uma estrutura conceitual para a modelagem de sistemas de gestão, no caso o BSC, que permite maior probabilidade de sucesso durante o processo de sua implantação. Finalmente o estudo contribui para a compreensão mais adequada dos fenômenos reais de mudança e institucionalização de sistemas de gestão a partir da estrutura conceitual proposta por Burns e Scapens (2000). Neste contexto fica evidenciado que a visão normativa é necessária, no sentido da adoção de melhores práticas de gestão, porém, a visão institucional é igualmente importante, no sentido de se assegurar a efetiva institucionalização do sistema idealizado.

\section{REFERÊNCIAS}

BAINES, A.; LANGFIELD-SMITH, K. Antecedents to management accounting change: astructural equation approach. Accounting, Organizations and Society, v. 28, n. 7, p. 675698, 2003.

BARLEY, S. R.; TOLBERT, P. S. Institutionalization and structuration: studying the links between action and institution. Organization Studies, n. 18, a.1, p. 93-117, 1997.

BRIGNALL, S. A contingent rationale for cost system design in services. Management Accounting Research, 8, p. 325-346, 1997.

BURNS, J. The dynamics of accounting change. Interplay between new practices, routines, institutions, power and politics. Accounting, Auditing \& Accountability Journal, 13(5), 566-596, 2000.

BURNS, J.; SCAPENS, R. W. Conceptualizing management accounting change: an institutional framework. Management Accounting Research, v. 11, p. 3-25, 2000.

CERVO, A. L., BERVIAN, P. A., DA SILVA, R. Metodologia Científica. $6^{\text {a }}$ ed. São Paulo: Pearson Prentice Hall, 2007, 
CREPALDI, S. A. Contabilidade Gerencial: Teoria e Prática. 6a ed. São Paulo: Atlas, 2012.

DUTRA, J. S. Competências: conceitos e instrumentos para a gestão de pessoas na empresa moderna. Atlas, 2004.

ECCLES, R. G. The Performance measurement manifesto. Harvard Business Review, v. 69, n. 1, p. 131-137, 1991.

ECCLES, R. G.; PYBURN, P. J. Creating a Comprehensive System to Measure Performance. Management Accounting, v.74, n.4, p.41, Oct 1992.

FEREIRA, A; OTLEY, D. The design and use of performance management systems: An extended framework for analysis. Management Accounting Research. n. 20, p. 263-282, 2009.

FERNANDES, B. H. R.; BERTON, L. H. Administração estratégica: da competência empreendedora à avaliação de desempenho. São Paulo: Saraiva, 2005.

FONSECA, V.; MACHADO-DA-SILVA, C. L. Conversação entre abordagens d estratégia em organizações: escolha estratégica, cognição e instituição. Organizações e Sociedade, v. 9, n. 25, p. 93-109, 2002.

FREZATTI, F. Orçamento empresarial: planejamento e controle gerencial. 4. ed. São Paulo: Atlas, 2007.

FRIGO, M. L. Strategy-focused performance measures. Strategic Finance, 84(3), 10-15, 2002.

HERRERO FILHO, E. Balanced Scorecard e a gestão estratégica: uma abordagem prática. Rio de Janeiro: Campus, 2005.

HODGSON, G. M. What are institutions?. Journal of Economic Issues, 40(1), 1-25, 1998.

HORNGREN, C. T. SUNDEM, G. L.; STRATTON, W. O. Contabilidade Gerencial. 12. ed. São Paulo: Prentice Hall, 2004.

HUMPHREY, C.; SCAPENS, R. W. Methodological themes: theories and case studies of organizational accounting practices: limitation or liberation?. Accounting, Auditing \& Accountability Journal, 9(4), 86-106, 1996.

KAPLAN, R. S.; NORTON, D. P. A estratégia em ação: balanced scorecard. Rio de Janeiro, editora Campus, 1997.

.Transforming the balanced scorecard from performance measurement to strategic management: part 1. Accounting Horizons, v.15, n.1, p.87, Mar. 2001.

. The strategy map: guide to aligning intangible assets. Strategy \& Leadership, v. 32, n. 5, p. 10, 2004. 
KENNERLEY, M.; NEELY, A. A framework of the factors affecting the evolution of performance measurement systems. International journal of operations \& production management, v. 22, n. 11, p. 1222-1245, 2002.

DE LIMA, C. R. M.; SOARES, T. C.; DE LIMA, M. A. Utilização do Balanced Scorecard em Instituições de Ensino Superior. Revista de Informação Contábil, v. 6, n. 3, 2012.

LUNKES, R. J.; SCHNORRENBERGER, D.; GASPARETTO, V.; CAPARICA, C. E. Implementação do Balanced Scorecard em uma Empresa de Software. Pensar Contábil, v. 11, n. 45 , p. 24-33, 2009.

MACHADO, M. J. C. V. Balanced Scorecard: an empirical study of small and medium size enterprises. RBGN Review of Business Management, v. 15, n. 46, p. 129-148, 2013.

MARTINS, T. S.; CRUZ, J. A. W.; CORSO, J. M. D. O impacto da implementação do balanced scorecard no desempenho financeiro. Revista Gestão e Planejamento, v. 12, n. 1, p. 61-73, 2011.

MARTINSONS, M.; DAVISON, R.; TSE, D. The balanced scorecard: a foundation for the strategic management of information systems. Decision support systems, v. 25, n. 1, p. 7188, 1999.

MERCHANT, K. A. Measuring general managers' performances: Market, accounting and combination-of-measures systems. Accouting, Auditing e Accountability Journal, v.19, n.6, p.893-917, Nov./Dec. 2006.

MOLL, J.; BURNS, J.; MAJOR, M. Institutional theory. in Hoque, Z. (Ed.), Methodological Issues in Accounting Research: Theories and Methods. Spiramus,183-205, 2006.

NIVEN, P. R.; Balanced Scorecard step by step: maximizing performance and maintaining results. New York: John Wiley \& Sons, Inc., 2002.

OLIVER, C. Sustainable competitive advantage: combining institutional and resource-based views. Strategic Management Journal. v. 18, n. 9, p. 697-713, 1997.

OLSON, E. M.; SLATER, S. F. The balanced scorecard, competitive strategy and performance. Business Horizons, v. 45, n. 3, p. 11-17, May/June 2002.

PRADO, L. J. Guia Balanced Scorecard, 2002. Disponível em:

$<$ http://www.secth.com.br/books/GUIA_Balance_Scorecard.pdf $>$. Acesso em 07 out. 2013.

PRIETO, V. C.; PEREIRA, F. L. A.; CARVALHO, M. M. D.; LAURINDO, F. J. B. Fatores críticos na implementação do Balanced Scorecard. Gestão e Produção, v. 13, n. 1, p. 81-92, 2006.

REIS, L. G. A influência do discurso no processo de mudança da contabilidade gerencial: Um estudo de caso sob o enfoque da teoria institucional. Tese de doutorado, Faculdade de 
Economia, Administração e Contabilidade, Universidade de São Paulo, São Paulo, SP, Brasil, 2008.

RICHARDSON, R. J. Pesquisa social: métodos e técnicas. 2. ed. São Paulo: Atlas, 1989.

ROCHA, A. C. B. Configuração de um sistema de avaliação de desempenho alicerçado no balanced scorecard para uma indústria de confecções de porte médio. Dissertação (Mestrado em Engenharia da Produção). Programa de Pós-Graduação em Engenharia da Produção, Universidade Federal de Santa Catarina, 2002.

ROCHA, I.; LAVARDA, C. E. F. Retrospectiva bibliográfica sobre o Balanced Scorecard (BSC) como instrumento de planejamento e controle nas empresas. Revista de

Contabilidade do Mestrado em Ciências Contábeis da UERJ, v. 16, n. 1, p. 19-34, 2011.

ROCHA, W.; GUERREIRO, R. Desenvolvimento de modelo conceitual de sistemas de custos - um enfoque institucional. RCO - Revista de Contabilidade e Organizações - FEARP/USP, v. 4, n. 8, p. 24-24, jan-abr 2010.

RUSSO, P. T.; PARISI, C.; MEGLIORINI, E.; ALMEIDA, C. B. D. Evidências de elementos de institucionalização do Balanced Scorecard na obra "A estratégia em ação": um olhar baseado na teoria institucional. Revista de Contabilidade e Finanças - USP, São Paulo, 23(58), 7-18, jan./fev./mar./abr, 2012.

SCAPENS, R. W. Researching management accounting practice: the role of case study methods. The British Accounting Review, 22(3), 259-281, 1990.

SCAPENS, R. W.; ROBERTS, J. Accounting and control: a case study of resistance to accounting change. Management Accounting Research, 4(1), 1-32, 1993.

SCHARMACH, A. L. da R. Gestão estratégica em instituições de ensino superior: as possibilidades do balanced scorecard na universidade do contestado. Dissertação de Mestrado apresentada ao Programa de Pós-Graduação Stricto Sensu em Administração do Centro de Ciências Sociais Aplicadas da Universidade Regional de Blumenau, 2010.

SCHIMIDT, S. R.; KIEMELE, M. J.; BERDINE, R. J. Knowledge-based management. Colorado Springs: Air Academy. Press \& Associates, LLC, p. 195, 1999.

SENGE, P. M. The fifth discipline, the art and practice of the learning organization. Performance Instruction, v. 30, n. 5, p. 37-37, 1991.

SIMÕES, A. M. D.; RODRIGUES, J. A. A abordagem da velha economia institucional na investigação em contabilidade e controle de gestão contributos teóricos.

Revista Iberoamericana de Contabilidad de Gestión - RIGC, 10(19), enero/junio. 2012.

SIMONS, R. Performance measurement and control systems for implementing strategy. Upper Saddle River: Prentice Hall, Inc., p. 348, 2000. 
SOIN, K.; SEAL, W.; CULLEN, J. ABC and organizational change: an institutional perspective. Management Accounting Research, v. 13, n. 2, p. 249-271, 2002.

SPESSATTO, G. Análise do processo de implantação do balanced scorecard nas maiores empresas da região sul do Brasil. Dissertação (Mestrado em Ciências Contábeis). Programa de Pós-Graduação em Ciências Contábeis, Universidade Regional de Blumenau, 2009.

YIN, R. K. Estudo de Caso: planejamento e métodos. Tradução de Daniel Grassi. $3^{\text {a }}$ ed. Porto Alegre: Bookman, 2010.

YUEN; S. M. Performance measurement and management of third party logistics: an organizational theory approach. Thesis (Doctoral of Philosophy) - Hong Kong Baptist University, 2006.

ZAGO, C. A.; ABREU, L. F.; GRZEBIELUCKAS, C.; BORNIA, A.C. Modelo de avaliação de desempenho logístico com base no Balanced Scorecard (BSC): proposta para uma pequena empresa. Revista da Micro e Pequena Empresa, v. 2, n. 1, p. 19-37, 2008. 